\title{
Assessment of expiratory flow limitation in chronic obstructive pulmonary disease: a new approach
}

\author{
R. Farré, D. Navajas
}

Patients with severe chronic obstructive pulmonary disease (COPD) usually experience expiratory flow limitation (EFL) during spontaneous breathing at rest [1]. As EFL reduces the effectiveness of expiration, it results in dynamic hyperinflation with consequent dyspnoea, which is one of the major complaints of patients with COPD. In these patients, the consequences of EFL are markedly increased during exercise [2]. Bearing in mind that EFL is a good predictor of dyspnoea in COPD patients $[3,4]$, simple methods for detecting EFL without perturbing normal breathing are of clinical interest. In 1995 Koulouris et al. [5] proposed a method to detect EFL based on the external application of a negative pressure at the mouth during tidal expiration (negative expiratory pressure (NEP)). EFL is detected by comparing the magnitude of expiratory flow before and after application of NEP: in case of EFL, the expiratory flow does not rise when increasing the driving pressure. More recently, NinANE et al. [6] proposed the estimation of EFL during spontaneous breathing with a similar approach. To increase the driving pressure during expiration, these authors applied a positive abdominal pressure by manual compression of the patient's abdominal wall. When compared with the NEP technique, this approach has the advantage of instrumental simplicity, but suffers from the drawbacks of applying abdominal pressure of uncontrolled magnitude and lack of automatisation. Both procedures can be applied during breathing at rest as well as during exercise [7,8].

In this issue of the European Respiratory Journal (ERJ), DELLACÀ et al. [9] present interesting novel data showing that the forced oscillation technique (FOT) can be useful for noninvasively detecting EFL during spontaneous breathing. The FOT, which was proposed in the 1950s [10], is based on applying a small-amplitude oscillation pressure at the mouth. Using the FOT the patient's respiratory mechanics can be determined by simply recording the oscillatory pressure and flow signals at the mouth, provided that the oscillation frequency is much higher than the breathing rate. The relationship between the pressure and the flow oscillation is the impedance of the respiratory system, which has two components: respiratory resistance ( $R \mathrm{rs})$ and reactance $(X \mathrm{rs})$. In a simple interpretation, $R$ rs is attributed to airways and tissue resistances, whereas $X \mathrm{rs}$ is determined by the inertial and compliant properties of the respiratory system. The rationale of using FOT for assessing EFL is that under this flow regime the forced oscillation applied at the mouth cannot reach the alveoli given the fact that the choke point shifts upstream of the lung during EFL. Therefore, the effective impedance viewed from the FOT device corresponds to only a

Unitat de Biofisica i Bioenginyeria, Facultat de Medicina, Universitat de Barcelona-IDIBAPS, Barcelona, Spain.

Correspondence: R. Farré, Unitat de Biofisica i Bioenginyeria, Facultat de Medicina, Universitat de Barcelona-IDIBAPS, Barcelona, Spain. Fax: 34 934024516. E-mail: rfarre@ub.edu fraction of the respiratory system: from the mouth to the choke point. According to this hypothesis, the associated decrease in the effective respiratory compliance results in a reduction of the measured $X \mathrm{rs}$ during expiration when compared with the non flow-limited inspiration.

The notion that phasic changes in $X$ rs during the breathing cycle could be an index of EFL is not new. This was suggested in 1993 by PESLIN et al. [11] when studying mechanically ventilated COPD patients, and the hypothesis was more specifically substantiated in a controlled analog model [12] and animal studies [13]. However, the usefulness of the FOT for detecting EFL in spontaneously breathing COPD patients was not studied in detail before the work of DELLACÀ et al. [9] published in this issue of the ERJ. These authors analysed the sensitivity and specificity of FOT to detect EFL in each individual breathing cycle by computing the phasic changes in $X$ rs within the cycle. As a reference technique to assess whether EFL was present or not in the same breathing cycle, DELlacÀ et al. [9] used the MEAD and WhitTENBERGER [14] method. This is based on the graphic analysis of the loop determined by the flow signal and by the resistive component of the transpulmonary pressure signal, which is measured by means of an oesophageal balloon.

The results reported by DELLACÀ et al. [9] strongly support their hypothesis that the FOT applied at a frequency of $5 \mathrm{~Hz}$ is a useful tool for detecting EFL in spontaneously breathing COPD patients. Specifically, the authors report that they were able to find thresholds for the indices representing the phasic changes in $X$ rs that resulted in a $100 \%$ sensitivity and specificity. Obviously, these excellent results, which were obtained from a research pilot study including a limited number of patients and controls, need to be further confirmed by more extensive studies. In this regard, a key issue is whether the thresholds found by the authors would keep their high sensitivity and specificity when applied to a wider sample of patients. However, according to the data and reasoning provided by the authors, it is plausible to expect further support for their conclusions when the technique is applied in future studies. Another major issue warranting future studies is to what extend the FOT allows determination of the part expiration under EFL. Moreover, the technique should be assessed during exercise, particularly to determine whether a FOT frequency of $5 \mathrm{~Hz}$ is sufficiently high to keep an acceptable signal-to-noise ratio given the expected increase in the patient's breathing rate. The promising results reported by DELLACÀ et al. [9] as regards the application of the FOT to assess EFL also warrant further studies applying this technique and the NEP method in the same patients to compare the practical advantages and disadvantages of both approaches.

Besides its specific interest as a technique to assess EFL, the work of DELLACÀ et al. [9] is also relevant to better clarify some issues on the interpretation of $R$ rs and $X$ rs values measured by the FOT in routine applications in patients who 
are potentially under EFL. As has been recently summarised by a European Respiratory Society Task Force report on the FOT [15], the Rrs values measured by this technique are useful to quantify airway obstruction in a variety of applications. However, the data from DellacÀ et al. [9] suggest that $X$ rs could be a more suitable index than $R$ rs to assess respiratory mechanics during EFL. The results of these authors also indicate $[12,13]$ that averaging the impedance data over the whole breathing cycle, which is the most common procedure [15], could result in data misinterpretation when FOT is applied in COPD patients. Accordingly, tracking the impedance data along the breathing cycle or, at least, separating inspiratory and expiratory data could improve the performance of FOT in evaluating the respiratory mechanics of COPD patients $[9,12,13]$.

It is worth noting that the approach proposed by DELLACÀ et al. [9] to assess EFL is readily applicable in routine. Indeed, it allows the automatic and continuous assessment of EFL based on FOT indices obtained by the simple application of a relatively low frequency $(5 \mathrm{~Hz})$ sinusoidal oscillation during spontaneous breathing. The advantage of such a simple FOT setting is that the proposed procedure can be applied not only in the lung function laboratory but in other situations where assessment of EFL could be of considerable interest. First, given that $5-\mathrm{Hz}$ sinusoidal oscillation can be easily incorporated into noninvasive mechanical ventilators [16], it could be possible to assess whether a ventilated patient is experiencing EFL and to modify the ventilator settings to improve ventilation. Second, it has been recently reported that a 5-Hz sinusoidal FOT can be implemented in a portable device [17] allowing the patient to self-assess their impedance at home [18]. Accordingly, application of the method proposed by Dellacì et al. [9] in this setting could allow an easy monitoring of EFL in COPD patients at home.

In conclusion, the research work of DELlaCÀ et al. [9] provides solid evidence that the forced oscillation technique is a simple procedure for detecting expiratory flow limitation during normal breathing. Future clinical studies should assess how the forced oscillation technique compares with other automatically applicable methods such as negative expiratory pressure, and should demonstrate the potential effectiveness of routine assessment of expiratory flow limitation in chronic obstructive pulmonary disease patients during spontaneous breathing at rest and during exercise.

\section{References}

1. Hyatt RE. The interrelationship of pressure, flow and volume during various respiratory maneuvers in normal and emphysematous patients. Am Rev Respir Dis 1961; 83: 676-683.

2. Marin JM, Carrizo SJ, Gascon M, Sanchez A, Gallego B, Celli BR. Inspiratory capacity, dynamic hyperinflation, breathlessness, and exercise performance during the 6-minute-walk test in chronic obstructive pulmonary disease. Am J Respir Crit Care Med 2001; 163: 1395-1399.

3. Eltayara L, Becklake MR, Volta CA, Milic-Emili J. Relationship between chronic dyspnea and expiratory flow limitation in patients with chronic obstructive pulmonary disease. Am J Respir Crit Care Med 1996; 154: 1726-1734.

4. Boni E, Corda L, Franchini D, et al. Volume effect and exertional dyspnoea after bronchodilator in patients with COPD with and without expiratory flow limitation at rest. Thorax 2002; 57: 528-532.

5. Koulouris NG, Valta $\mathrm{P}$, Lavoie A, et al. A simple method to detect expiratory flow limitation during spontaneous breathing. Eur Respir J 1995; 8: 306-313.

6. Ninane V, Leduc D, Kafi SA, Nasser M, Houa M, Sergysels R. Detection of expiratory flow limitation by manual compression of the abdominal wall. Am J Respir Crit Care Med 2001; 163: $1326-1330$

7. Koulouris NG, Dimopoulou I, Valta P, Finkelstein R, Cosio MG, Milic-Emili J. Detection of expiratory flow limitation during exercise in COPD patients. J Appl Physiol 1997; 82: 723-731.

8. Abdel Kafi S, Serste T, Leduc D, Sergysels R, Ninane V. Expiratory flow limitation during exercise in COPD: detection by manual compression of the abdominal wall. Eur Respir J 2002; 19: 919-927.

9. Dellacà RL, Santus $\mathrm{P}$, Aliverti A, et al. Detection of expiratory flow limitation in COPD using the forced oscillation technique. Eur Respir J 2004; 23: 232-240.

10. DuBois AB, Brody AW, Lewis DH, Burgess BF. Oscillation mechanics of lungs and chest in man. $J$ Appl Physiol 1956; 8: 587-594.

11. Peslin R, Felicio da Silva J, Duvivier C, Chabot F Respiratory mechanics studied by forced oscillations during artificial ventilation. Eur Respir J 1993; 6: 772-784.

12. Peslin R, Farre R, Rotger M, Navajas D. Effect of expiratory flow limitation on respiratory mechanical impedance: a model study. J Appl Physiol 1996; 81: 2399-2406.

13. Vassiliou M, Peslin R, Saunier C, Duvivier C. Expiratory flow limitation during mechanical ventilation detected by the forced oscillation method. Eur Respir J 1996; 9: 779-786.

14. Mead J, Whittenberger JL. Physical properties of human lungs measured during spontaneous respiration. $J$ Appl Physiol 1953; 5: 779-796.

15. Oostveen E, MacLeod D, Lorino $\mathrm{H}$, et al. The forced oscillation technique in clinical practice: methodology, recommendations and future developments. Eur Respir $J$ 2003; 22: 1026-1041.

16. Farre R, Mancini M, Rotger M, Ferrer M, Roca J, Navajas D. Oscillatory resistance measured during noninvasive proportional assist ventilation. Am J Respir Crit Care Med 2001; 164: 790-794.

17. Rigau J, Farre R, Roca J, Marco S, Herms A, Navajas D. A portable forced oscillation device for respiratory home monitoring. Eur Respir J 2002; 19: 146-150.

18. Rigau J, Burgos F, Hernandez C, Roca J, Navajas D, Farre R. Unsupervised self-testing of airway obstruction by forced oscillation at the patient's home. Eur Respir J 2003; 22: $668-671$ 